\title{
A global compactness result for a critical nonlinear Choquard equation in $\mathbb{R}^{N}$
}

Wangcheng Huang ${ }^{1}$, Wei Long ${ }^{1 *}$, Aliang Xia ${ }^{1}$ and Xiongjun Zheng ${ }^{1}$

"Correspondence:

Iwhope@jxnu.edu.cn

'Department of Mathematics, Jiangxi Normal University,

Nanchang, P.R. China

The Author(s) 2019. This article is distributed under the terms of the Creative Commons Attribution 4.0 International License (http://creativecommons.org/licenses/by/4.0/), which permits unrestricted use, distribution, and reproduction in any medium, provided you give appropriate credit to the original author(s) and the source, provide a link to the Creative Commons license, and indicate if changes were made.

\section{Introduction}

In this article, we consider the following nonlinear Choquard problem in $\mathbb{R}^{N}$ :

$$
-\Delta u+a(x) u=\left(|x|^{-\mu} *|u|^{2_{\mu}^{*}}\right)|u|^{2_{\mu}^{*}-2} u+q(x)|u|^{p-1} u, \quad u \in H^{1}\left(\mathbb{R}^{N}\right)
$$

where $2_{\mu}^{*}=\frac{2 N-\mu}{N-2}$ is the critical exponent in the sense of the Hardy-Littlewood-Sobolev inequality, $0<\mu<N, 1<p<2^{*}-1=\frac{N+2}{N-2}$ and $N \geq 4$.

This nonlocal elliptic equation is closely related to the nonlinear Choquard equation

$$
-\Delta u+V(x) u=\left(|x|^{-\mu} *|u|^{p}\right)|u|^{p-2} u \quad \text { in } \mathbb{R}^{3} .
$$

Different from the fractional Laplacian where the pseudo-differential operator causes the nonlocal phenomena, for the Choquard equation the nonlocal term appears in the nonlinearity and influences the equation greatly. For $p=2$ and $\mu=1$, it goes back to the description of the quantum theory of a polaron at rest by Pekar in 1954 [25] and the modeling of an electron trapped in its own hole in 1976 in the work of Choquard, as a certain approximation to Hartree-Fock theory of one-component plasma [14]. In some particular cases, this equation is also known as the Schrödinger-Newton equation, which was 
introduced by Penrose in his discussion of the self-gravitational collapse of a quantum mechanical wave function [26]. The existence and qualitative properties of solutions of (1.2) have been widely studied in the last decades, cf. [1, 2, 8, 12, 14, 16, 18, 20-23] and the references therein.

By the Hardy-Littlewood-Sobolev inequality (see [15]), the integral

$$
\int_{\mathbb{R}^{N}} \int_{\mathbb{R}^{N}} \frac{|u(x)|^{q}|u(y)|^{q}}{|x-y|^{\mu}} d x d y
$$

is well defined provided $|u|^{q} \in L^{t}\left(\mathbb{R}^{N}\right)$ for some $t>1$ satisfying

$$
\frac{2}{t}+\frac{\mu}{N}=2
$$

Therefore, by a Sobolev embedding, for some $u \in H^{1}\left(\mathbb{R}^{N}\right)$, we have

$$
2 \leq t q \leq \frac{2 N}{N-2}
$$

that is,

$$
\frac{2 N-\mu}{N} \leq q \leq \frac{2 N-\mu}{N-2} .
$$

So, we call $\frac{2 N-\mu}{N}$ the lower critical exponent and $2_{\mu}^{*}=\frac{2 N-\mu}{N-2}$ is the upper critical exponent in the sense of the Hardy-Littlewood-Sobolev inequality.

In [23], Moroz and Van Schaftingen considered the existence and nonexistence of solutions for nonlinear Choquard equation (1.2) in $\mathbb{R}^{N}$ with lower critical exponent. In [11], Gao and Yang studied the Brezis-Nirenberg type problem for a nonlinear Choquard equation with upper critical exponent in a bounded domain, that is,

$$
-\Delta u=\left(|x|^{-\mu} *|u|^{2_{\mu}^{*}}\right)|u|^{2_{\mu}^{*}-2} u+\lambda u \quad \text { in } \Omega,
$$

where $\Omega$ is a bounded domain of $\mathbb{R}^{N}$ with Lipschitz boundary, $\lambda$ is a real parameter. The main difficulty of solving this problem by critical point theory is the embedding losing compactness, then the so-called Palais-Smale condition is generally not satisfied for the related functional. It is shown in [11] that the obstacle of compactness is related to the function

$$
U(x)=C\left(\frac{b}{b^{2}+|x-a|^{2}}\right)^{\frac{N-2}{2}},
$$

where $C>0$ is a fixed constant, $a \in \mathbb{R}^{N}$ and $b \in(0, \infty)$ are parameters. In [11], the authors also proved that $U$ is the minimizer of the problem

$$
S_{H, L}=\inf _{u \in D^{1,2}\left(\mathbb{R}^{N}\right) \backslash\{0\}} \frac{\int_{\mathbb{R}^{N}}|\nabla u(x)|^{2} d x}{\left(\int_{\mathbb{R}^{N}} \int_{\mathbb{R}^{N}} \frac{|u(x)|^{2_{\mu}^{*}}\left|u(y)^{2}\right|^{*}}{|x-y|^{\mu}} d x d y\right)^{\frac{1}{2 *}}},
$$

and $U$ solves

$$
-\Delta u=\left(|x|^{-\mu} *|u|^{2^{*}}\right)|u|^{2_{\mu}^{*}-2} u \text { in } \mathbb{R}^{N} .
$$


It is proved in [11] that the associated functional of problem (1.3)

$$
I(u)=\frac{1}{2} \int_{\mathbb{R}^{N}}\left(|\nabla u|^{2}-\lambda u^{2}\right) d x-\frac{1}{2 \cdot 2_{\mu}^{*}} \int_{\mathbb{R}^{N}} \int_{\mathbb{R}^{N}} \frac{|u(x)|^{2_{\mu}^{*}}|u(y)|^{2_{\mu}^{*}}}{|x-y|^{\mu}} d x d y
$$

may lose the compactness in

$$
\left[\frac{N+2-\mu}{4 N-2 \mu} S_{H, L}^{\frac{2 N-\mu}{N-\mu+2}},+\infty\right)
$$

and this is fully caused by the solution of the "limit equation" (1.6). We also refer the reader to $[7,19,24,28,29]$ for related work.

In this article, we study the global compactness of critical nonlinear Choquard equation in $\mathbb{R}^{N}$; no such results for the problem can be found in the literature as far as we know.

A global compact result for a semilinear elliptic problem with critical Sobolev nonlinearities on the bounded domains was obtained by Struwe [31] and Lions [17]. It was known that the sub-level which makes the Palais-Smale conditions hold is determined by a compactness result [17, 31]. Pierrotti and Terracini [27] studied a class of critical elliptic equations with Neumann boundary conditions through a compact analysis. Ye and $\mathrm{Yu}$ [34] considered critical elliptic equations with lower order terms. Cao and Peng [6], Jin and Deng [13] got a global compact result for a class of critical elliptic equations with critical Sobolev and Hardy exponents in bounded domains and in $\mathbb{R}^{N}$, respectively.

Inspired by the work of $[6,13,17,31,34]$, we study the global compactness result of problem (1.1) in this paper. We make the following assumptions:

(A1) $a(x), q(x)$ are two positive continuous functions, such that inf $f_{x \in \mathbb{R}^{N}} a(x)>a_{0}>0$ and $q(x) \in L^{\infty}\left(\mathbb{R}^{N}\right)$.

(A2) $\lim _{|x| \rightarrow \infty} a(x)=\bar{a}, \lim _{|x| \rightarrow \infty} q(x)=\bar{q}$.

In the following, we assume that $\bar{a}=\bar{q}=1$ without of loss generality. The functional corresponding to $(1.1)$ is

$$
\begin{aligned}
J(u)= & \frac{1}{2} \int_{\mathbb{R}^{N}}|\nabla u(x)|^{2}+a(x)|u(x)|^{2} d x-\frac{1}{2 \cdot 2_{\mu}^{*}} \int_{\mathbb{R}^{N}} \int_{\mathbb{R}^{N}} \frac{|u(x)|^{2_{\mu}^{*}}|u(y)|^{2_{\mu}^{*}}}{|x-y|^{\mu}} d x d y \\
& -\frac{1}{p+1} \int_{\mathbb{R}^{N}} q(x)|u(x)|^{p+1} d x .
\end{aligned}
$$

We will show that the loss of compactness of $J$ is caused both by the critical exponent and the unbounded domain. To state the result more precisely, it is convenient to introduce the problems "at infinity" - the first one is problem (1.6) and the second is

$$
-\Delta u+u=\left(|x|^{-\mu} *|u|^{2_{\mu}^{*}}\right)|u|^{2_{\mu}^{*}-2} u+|u|^{p-1} u, \quad u \in H^{1}\left(\mathbb{R}^{N}\right) .
$$

Let

$$
\begin{aligned}
J_{\infty}(u)= & \frac{1}{2} \int_{\mathbb{R}^{N}}|\nabla u(x)|^{2}+|u(x)|^{2} d x-\frac{1}{2 \cdot 2_{\mu}^{*}} \int_{\mathbb{R}^{N}} \int_{\mathbb{R}^{N}} \frac{|u(x)|^{2_{\mu}^{*}}|u(y)|^{2_{\mu}^{*}}}{|x-y|^{\mu}} d x d y \\
& -\frac{1}{p+1} \int_{\mathbb{R}^{N}}|u(x)|^{p+1} d x,
\end{aligned}
$$


for $u \in H^{1}\left(\mathbb{R}^{N}\right)$ and

$$
I_{\infty}(u)=\frac{1}{2} \int_{\mathbb{R}^{N}}|\nabla u|^{2} d x-\frac{1}{2 \cdot 2_{\mu}^{*}} \int_{\mathbb{R}^{N}} \int_{\mathbb{R}^{N}} \frac{|u(x)|^{2_{\mu}^{*}}|u(y)|^{2_{\mu}^{*}}}{|x-y|^{\mu}} d x d y
$$

for $u \in D^{1,2}\left(\mathbb{R}^{N}\right)$.

Our main result is the following.

Theorem 1.1 Let $\left\{u_{n}\right\} \subset H^{1}\left(\mathbb{R}^{N}\right)$ be a sequence such that $J\left(u_{n}\right) \rightarrow c$ and $J^{\prime}\left(u_{n}\right) \rightarrow 0$, then there are sequences of points $\left\{y_{n}^{j}\right\}\left(1 \leq j \leq k_{1}\right), x_{n}^{j}\left(1 \leq j \leq k_{2}\right)$, sequences of numbers $\left\{R_{n}^{j}\right\}$ $\left(1 \leq j \leq k_{2}\right)$, sequences of functions $\left\{u_{n}^{j}\right\}\left(1 \leq j \leq k_{1}\right),\left\{v_{n}^{j}\right\}\left(1 \leq j \leq k_{2}\right)$, such that, for a subsequence of $\left\{u_{n}\right\}$, still denoted by $\left\{u_{n}\right\}$,

(i) $u_{n}(x)=u_{n}^{0}(x)+\sum_{1}^{k_{1}} u_{n}^{j}\left(x-y_{n}^{j}\right)+\sum_{1}^{k_{2}}\left(R_{n}^{j}\right)^{\frac{N-2}{2}} v_{n}^{j}\left(R_{n}^{j} x-x_{n}^{j}\right)$;

(ii) $u_{n}^{0} \rightarrow u^{0}(x)$ as $n \rightarrow \infty$ strongly in $H^{1}\left(\mathbb{R}^{N}\right)$;

(iii) $u_{n}^{j} \rightarrow u^{j}$ as $n \rightarrow \infty$ strongly in $H^{1}\left(\mathbb{R}^{N}\right)$ for $1 \leq j \leq k_{1}, v_{n}^{j}-\left(R_{n}^{j}\right)^{\frac{N-2}{2}} v^{j}\left(R_{n}^{j} x-x_{n}^{j}\right) \rightarrow 0$

as $n \rightarrow \infty$ strongly in $H^{1}\left(\mathbb{R}^{N}\right)$ for $1 \leq j \leq k_{2}$,

where $u^{0}$ is a solution of $(1.1), u^{j}\left(1 \leq j \leq k_{1}\right)$ are solutions of $(1.9)$ and $v^{j}\left(1 \leq j \leq k_{2}\right)$ are solutions of (1.6).

Moreover, as $m \rightarrow \infty$,

$$
\begin{aligned}
& \left\|u_{n}\right\|_{H^{1}\left(\mathbb{R}^{N}\right)}^{2} \rightarrow\left\|u^{0}\right\|_{H^{1}\left(\mathbb{R}^{N}\right)}^{2}+\sum_{j=1}^{k_{1}}\left\|u^{j}\right\|_{H^{1}\left(\mathbb{R}^{N}\right)}^{2}+\sum_{j=1}^{k_{2}}\left\|v^{j}\right\|_{D^{1,2}\left(\mathbb{R}^{N}\right)^{\prime}}^{2}, \\
& J\left(u_{n}\right) \rightarrow J\left(u^{0}\right)+\sum_{j=1}^{k_{1}} J_{\infty}\left(u^{j}\right)+\sum_{j=1}^{k_{2}} I_{\infty}\left(v^{j}\right) .
\end{aligned}
$$

Using the above compact results and some delicate analysis, we have the following corollary.

Corollary 1.2 The functional J satisfies the $(P S)_{c}$ for $c \in\left(0, c_{\infty}\right)$, where $c_{\infty}$ is the least energy of $J_{\infty}$.

Finally, applying the mountain pass theorem (cf. [3]), we can get the following existence result of problem (1.1).

Corollary 1.3 Assume $a(x) \leq \bar{a}$ and $a(x)<\bar{a}$ in a positive measure set or $q(x) \geq \bar{q}$ and $q(x)>\bar{q}$ in a positive measure set, then problem (1.1) has at least one solution.

This paper is organized as follows. In Sect. 2, we get an existence result of problem (1.9) in $H_{r}^{1}\left(\mathbb{R}^{N}\right)$. We prove our main results in Sect. 3.

\section{Existence results of problem (1.9)}

In order to obtain our results, we first need to show the existence of solutions to equation (1.9). In this section, we prove that there exists at least one solution to Eq. (1.9) via the 
mountain pass theorem in a radially symmetry Sobolev space, that is,

$$
H_{r}^{1}\left(\mathbb{R}^{N}\right)=\left\{u \in H^{1}\left(\mathbb{R}^{N}\right): u(x)=u(|x|)\right\} .
$$

More generally, we consider the following equation:

$$
-\Delta u+u=\left(|x|^{-\mu} *|u|^{2_{\mu}^{*}}\right)|u|^{2_{\mu}^{*}-2} u+f(u), \quad u \in H^{1}\left(\mathbb{R}^{N}\right),
$$

with $f$ satisfying:

$\left(f_{1}\right) f(t) \in C^{2}\left(\mathbb{R}^{1}\right), \lim _{t \rightarrow 0} \frac{f(t)}{t}=0, \lim _{t \rightarrow \infty} \frac{f(t)}{t^{2^{*}-1}}=0 ;$

$\left(f_{2}\right)$ there exists an $\varepsilon \geq 0$ which is small enough, such that

$$
t(f(t))^{\prime} \geq(1+\varepsilon) f(t) \geq 0
$$

for all $t \geq 0$;

$\left(f_{3}\right) f(t)$ is odd.

Remark 2.1 Assumptions $\left(f_{1}\right)-\left(f_{3}\right)$ are introduced by Deng, Guo and Wang in [9] which studied the nodal solutions for the p-Laplacian.

The variational functional corresponding to $(2.1)$ is

$$
\begin{aligned}
\Phi(u)= & \frac{1}{2} \int_{\mathbb{R}^{N}}|\nabla u(x)|^{2}+|u(x)|^{2} d x-\frac{1}{2 \cdot 2_{\mu}^{*}} \int_{\mathbb{R}^{N}} \int_{\mathbb{R}^{N}} \frac{|u(x)|^{2^{*}}|u(y)|^{2_{\mu}^{*}}}{|x-y|^{\mu}} d x d y \\
& -\int_{\mathbb{R}^{N}} F(u) d x,
\end{aligned}
$$

where

$$
F(u)=\int_{0}^{u} f(t) d t
$$

By adapting the mountain pass theorem to (2.3), we can prove the following.

Theorem 2.2 Let $f(t)$ satisfy $\left(f_{1}\right)-\left(f_{3}\right)$, then (2.1) possesses a nontrivial solution $w \in$ $H_{r}^{1}\left(\mathbb{R}^{N}\right)$ such that

$$
\Phi(u)<\frac{N+2-\mu}{4 N-2 \mu} S_{H, L}^{\frac{2 N-\mu}{N-\mu+2}}
$$

provided $N \geq 4$.

To prove the (PS) condition, we need two key lemmas.

Lemma 2.3 ([33], Proposition 5.4.7) Let $N \geq 3,1<q<\infty$ and $\left\{u_{n}\right\}$ is a bounded sequence in $L^{q}\left(\mathbb{R}^{N}\right)$. If $u_{n} \rightarrow$ u a.e. in $\mathbb{R}^{N}$ as $n \rightarrow \infty$, then $u_{n} \rightarrow$ u weakly in $L^{q}\left(\mathbb{R}^{N}\right)$ as $n \rightarrow \infty$. 
Lemma 2.4 ([11], Lemma 2.2) Let $N \geq 3$ and $0<\mu<N$. If $\left\{u_{n}\right\}$ is a bounded sequence in $L^{\frac{2 N}{N-2}}\left(\mathbb{R}^{N}\right)$ such that $u_{n} \rightarrow$ u a.e. in $\mathbb{R}^{N}$ as $n \rightarrow \infty$, then

$$
\begin{aligned}
& \int_{\mathbb{R}^{N}}\left(|x|^{-\mu} *\left|u_{n}\right|^{2_{\mu}^{*}}\right)\left|u_{n}\right|^{2_{\mu}^{*}} d x-\int_{\mathbb{R}^{N}}\left(|x|^{-\mu} *\left|u_{n}-u\right|^{2_{\mu}^{*}}\right)\left|u_{n}-u\right|^{2_{\mu}^{*}} d x \\
& \rightarrow \int_{\mathbb{R}^{N}}\left(|x|^{-\mu} *|u|^{2_{\mu}^{*}}\right)|u|^{2_{\mu}^{*}} d x
\end{aligned}
$$

as $n \rightarrow \infty$.

In order to get a critical point of (2.3), we need some lemmas as follows.

Lemma 2.5 Let $\left(f_{1}\right)-\left(f_{3}\right)$ hold. If

$$
c \in\left(0, \frac{N+2-\mu}{4 N-2 \mu} S_{H, L}^{\frac{2 N-\mu}{N-\mu+2}}\right)
$$

then $\Phi(u)$ satisfies $(P S)_{c}$ condition.

Proof Let $\left\{u_{j}\right\}_{j \geq 1} \subset H_{r}^{1}\left(\mathbb{R}^{N}\right)$ be a $(P S)_{c}$ sequence, then, by a similar argument to Lemma 2.2 in [9] and Lemma 2.4 in [11], we know $\left\{u_{j}\right\}_{j \geq 1}$ is bounded in $H_{r}^{1}\left(\mathbb{R}^{N}\right)$. Thus by subtracting a subsequence of $\left\{u_{j}\right\}_{j \geq 1}$, still denoted by $\left\{u_{j}\right\}$, we have $u_{j} \rightarrow u$ weakly in $H_{r}^{1}\left(\mathbb{R}^{N}\right)$ as $j \rightarrow \infty$. By a Sobolev embedding, we know $u_{j} \rightarrow u$ weakly in $L^{2^{*}}\left(\mathbb{R}^{N}\right)$ as $j \rightarrow \infty$. Then

$$
\left|u_{j}\right|^{2_{\mu}^{*}} \rightarrow|u|^{2_{\mu}^{*}} \quad \text { weakly in } L^{\frac{2 N}{2 N-\mu}}\left(\mathbb{R}^{N}\right)
$$

as $j \rightarrow \infty$. By the Hardy-Littlewood-Sobolev inequality, we know that

$$
|x|^{-\mu} *\left|u_{j}\right|^{2_{\mu}^{*}} \rightarrow|x|^{-\mu} *|u|^{2_{\mu}^{*}} \quad \text { weakly in } L^{\frac{2 N}{\mu}}\left(\mathbb{R}^{N}\right)
$$

as $j \rightarrow \infty$. Combining with the fact

$$
\left|u_{j}\right|^{2_{\mu}^{*}-2} u_{j} \rightarrow|u|^{2_{\mu}^{*}-2} u \quad \text { weakly in } L^{\frac{2 N}{N-\mu+2}}\left(\mathbb{R}^{N}\right)
$$

as $j \rightarrow \infty$, we have

$$
\left(|x|^{-\mu} *\left|u_{j}\right|^{2_{\mu}^{*}}\right)\left|u_{j}\right|^{2_{\mu}^{*}-2} u_{j} \rightarrow\left(|x|^{-\mu} *|u|^{2_{\mu}^{*}}\right)|u|^{2_{\mu}^{*}-2} u \quad \text { weakly in } L^{\frac{2 N}{N+2}}\left(\mathbb{R}^{N}\right)
$$

as $j \rightarrow \infty$.

On the other hand, by Strauss' lemma (Lemma 2.1 in [30]), one can easily deduce that

$$
\begin{aligned}
& F\left(u_{j}\right) \rightarrow F(u) \quad \text { strongly in } L^{1}\left(\mathbb{R}^{N}\right), \\
& f\left(u_{j}\right) u_{j} \rightarrow f(u) u \quad \text { strongly in } L^{1}\left(\mathbb{R}^{N}\right) .
\end{aligned}
$$


Since $\Phi^{\prime}\left(u_{j}\right) \rightarrow 0$

$$
\begin{aligned}
& \int_{\mathbb{R}^{N}}(\nabla u \nabla \phi+u \phi) d x-\int_{\mathbb{R}^{N}} \int_{\mathbb{R}^{N}} \frac{|u(x)|^{2_{\mu}^{*}}|u(y)|^{2_{\mu}^{*}-2} u(y) \phi(y)}{|x-y|^{\mu}} d x d y \\
& -\int_{\mathbb{R}^{N}} f(u) \phi d x=0
\end{aligned}
$$

for any $\phi \in C_{0}^{\infty}\left(\mathbb{R}^{N}\right)$, which means $u$ is a weak solution of problem (2.1).

By $\left(f_{2}\right)$, we have

$$
F(u) \leq \frac{u f(u)}{2+\varepsilon}
$$

Combining this and (2.7) with $\phi=u$, then

$$
\begin{aligned}
\Phi(u)= & \left(\frac{1}{2}-\frac{1}{2 \cdot 2_{\mu}^{*}}\right) \int_{\mathbb{R}^{N}} \int_{\mathbb{R}^{N}} \frac{|u(x)|^{2_{\mu}^{*}}|u(y)|^{2_{\mu}^{*}}}{|x-y|^{\mu}} d x d y \\
& +\frac{1}{2} \int_{\mathbb{R}^{N}} f(u) u d x-\int_{\mathbb{R}^{N}} F(u) d x \\
\geq & 0 .
\end{aligned}
$$

Since $\left\{u_{j}\right\}$ is a $(P S)_{c}$ sequence, $\left\{u_{j}\right\}$ is bounded and thus (2.5)-(2.6) hold, we have

$$
\frac{1}{2}\left\|u_{j}\right\|_{H^{1}\left(\mathbb{R}^{N}\right)}^{2}-\frac{1}{2 \cdot 2_{\mu}^{*}} \int_{\mathbb{R}^{N}} \int_{\mathbb{R}^{N}} \frac{\left|u_{j}(x)\right|^{2_{\mu}^{*}}\left|u_{j}(y)\right|^{2_{\mu}^{*}}}{|x-y|^{\mu}} d x d y-\int_{\mathbb{R}^{N}} F(u) d x=c+o(1)
$$

and

$$
\left\|u_{j}\right\|_{H^{1}\left(\mathbb{R}^{N}\right)}^{2}-\int_{\mathbb{R}^{N}} \int_{\mathbb{R}^{N}} \frac{\left|u_{j}(x)\right|^{2_{\mu}^{*}}\left|u_{j}(y)\right|^{2_{\mu}^{*}}}{|x-y|^{\mu}} d x d y-\int_{\mathbb{R}^{N}} f(u) u d x=o(1) .
$$

Set $v_{j}=u_{j}-u$. By the Brezis-Lieb lemma (see [5]) and Lemma 2.4, we infer that

$$
\Phi(u)+\frac{1}{2}\left\|v_{j}\right\|_{H^{1}\left(\mathbb{R}^{N}\right)}^{2}-\frac{1}{2 \cdot 2_{\mu}^{*}} \int_{\mathbb{R}^{N}} \int_{\mathbb{R}^{N}} \frac{\left|v_{j}(x)\right|^{2_{\mu}^{*}}\left|v_{j}(y)\right|^{2_{\mu}^{*}}}{|x-y|^{\mu}} d x d y=c+o(1)
$$

and

$$
\left\|v_{j}\right\|_{H^{1}\left(\mathbb{R}^{N}\right)}^{2}-\int_{\mathbb{R}^{N}} \int_{\mathbb{R}^{N}} \frac{\left|v_{j}(x)\right|^{2_{\mu}^{*}}\left|v_{j}(y)\right|^{2_{\mu}^{*}}}{|x-y|^{\mu}} d x d y=o(1)
$$

Without loss of generality, we may assume that

$$
\lim _{j \rightarrow \infty}\left\|v_{j}\right\|_{H^{1}\left(\mathbb{R}^{N}\right)}^{2}=k
$$

Then by (2.10) we get

$$
\lim _{j \rightarrow \infty} \int_{\mathbb{R}^{N}} \int_{\mathbb{R}^{N}} \frac{\left|v_{j}(x)\right|^{2_{\mu}^{*}}\left|v_{j}(y)\right|^{2_{\mu}^{*}}}{|x-y|^{\mu}} d x d y=k
$$


By (1.5),

$$
\int_{\mathbb{R}^{N}}\left|\nabla v_{j}\right|^{2} d x \geq S_{H, L}\left(\int_{\mathbb{R}^{N}} \int_{\mathbb{R}^{N}} \frac{\left|v_{j}(x)\right|^{2_{\mu}^{*}}\left|v_{j}(y)\right|^{2_{\mu}^{*}}}{|x-y|^{\mu}} d x d y\right)^{\frac{1}{2_{\mu}^{*}}}
$$

for all $j$. So we have

$$
\left\|v_{j}\right\|_{H^{1}\left(\mathbb{R}^{N}\right)}^{2} \geq S_{H, L}\left(\int_{\mathbb{R}^{N}} \int_{\mathbb{R}^{N}} \frac{\left|v_{j}(x)\right|^{2_{\mu}^{*}}\left|v_{j}(y)\right|^{2_{\mu}^{*}}}{|x-y|^{\mu}} d x d y\right)^{\frac{1}{2_{\mu}^{*}}},
$$

for all $j$. Then, by taking $j \rightarrow \infty$, we get

$$
k \geq S_{H, L} k^{\frac{1}{2 *}} .
$$

If $k>0$, it follows from (2.11) that $k \geq S_{H, L}^{\frac{2 N-\mu}{N-\mu+2}}$. By (2.9) we get

$$
\Phi(u)=c-\frac{N+2-\mu}{4 N-2 \mu} S_{H, L}^{\frac{2 N-\mu}{N-\mu+2}}<0,
$$

this contradicts (2.8). Thus $k=0$. By definition of $v_{j}$, we conclude that $\Phi(u)$ satisfies the $(P S)_{c}$ condition. This completes the proof.

By Lemma 2.5 and the mountain pass theorem, one can easily verify the following lemma.

Lemma 2.6 Let $\left(f_{1}\right)-\left(f_{2}\right)$ hold. Suppose that there exists $u_{0} \in H_{r}^{1}\left(\mathbb{R}^{N}\right), u_{0} \neq 0$ such that

$$
\sup _{t \geq 0} \Phi\left(t u_{0}\right)<\frac{N+2-\mu}{4 N-2 \mu} S_{H, L}^{\frac{2 N-\mu}{N-\mu+2}}
$$

then problem (2.1) possesses at least one nontrivial weak solution.

In the following discussion we will prove that (2.12) naturally holds for $N \geq 4$.

Lemma 2.7 Let $\left(f_{1}\right)-\left(f_{2}\right)$ hold. Then there exists $u_{0} \in H_{r}^{1}\left(\mathbb{R}^{N}\right) \backslash\{0\}$, such that (2.12) holds for $N \geq 4$.

Proof Recall function $U$ given in (1.4) which is minimizer for $S_{H, L}$. Let $\varphi \in C_{0}^{\infty}\left(\mathbb{R}^{N}\right) \cap$ $H_{r}^{1}\left(\mathbb{R}^{N}\right)$ be a cut-off function such that

$$
\varphi(x)= \begin{cases}1 & \text { for }|x| \leq \rho \\ 0 \leq \varphi(x) \leq 1 & \text { for } \rho<|x|<2 \rho \\ 0 & \text { for }|x| \geq 2 \rho .\end{cases}
$$

We define, for $\varepsilon>0$,

$$
\begin{aligned}
& U_{\varepsilon}(x):=\varepsilon^{\frac{2-N}{2}} U\left(\frac{x}{\varepsilon}\right), \\
& u_{\varepsilon}(x):=\varphi(x) U_{\varepsilon}(x) .
\end{aligned}
$$


We prove this lemma by several steps. Firstly, by a similar argument to Lemma 3.5 in [9], for $\varepsilon$ small enough, there exists a constant $t_{\varepsilon}>0$ such that

$$
\Phi\left(t_{\varepsilon} u_{\varepsilon}\right)=\sup _{t \geq 0} \Phi\left(t u_{\varepsilon}\right)
$$

and

$$
0<C_{1}<t_{\varepsilon}<C_{2}<\infty
$$

where $C_{1}, C_{2}$ are positive constants independent of $\varepsilon$.

On the other hand, from [11], we know that

$$
\begin{aligned}
& \int_{\mathbb{R}^{N}}\left|\nabla u_{\varepsilon}\right|^{2} d x=C(N, \mu)^{\frac{N-2}{2 N-\mu} \cdot \frac{N}{2}} S_{H, L}^{\frac{N}{2}}+O\left(\varepsilon^{N-2}\right), \\
& \int_{\mathbb{R}^{N}}\left|u_{\varepsilon}\right|^{2} d x= \begin{cases}d \varepsilon^{2}|\log \varepsilon|+O\left(\varepsilon^{2}\right) & \text { if } N=4, \\
d \varepsilon^{2}+O\left(\varepsilon^{N-2}\right) & \text { if } N \geq 5,\end{cases} \\
& \int_{\mathbb{R}^{N}} \int_{\mathbb{R}^{N}} \frac{\left|u_{\varepsilon}(x)\right|^{2_{\mu}^{*}}\left|u_{\varepsilon}(y)\right|^{2_{\mu}^{*}}}{|x-y|^{\mu}} d x d y \leq C(N, \mu)^{\frac{N}{2}} S_{H, L}^{\frac{2 N-\mu}{2}}+O\left(\varepsilon^{N-\frac{\mu}{2}}\right),
\end{aligned}
$$

and

$$
\int_{\mathbb{R}^{N}} \int_{\mathbb{R}^{N}} \frac{\left|u_{\varepsilon}(x)\right|^{2_{\mu}^{*}}\left|u_{\varepsilon}(y)\right|^{2_{\mu}^{*}}}{|x-y|^{\mu}} d x d y \geq C(N, \mu)^{\frac{N}{2}} S_{H, L}^{\frac{2 N-\mu}{2}}-O\left(\varepsilon^{N-\frac{\mu}{2}}\right),
$$

where $C(N, \mu), d$ are positive constants. Therefore,

$$
\begin{aligned}
\Phi\left(t_{\varepsilon} u_{\varepsilon}\right) \leq & \max _{t \geq 0}\left\{\frac{t^{2}}{2} \int_{\mathbb{R}^{N}}\left|\nabla u_{\varepsilon}\right|^{2} d x-\frac{t^{2 \cdot 2_{\mu}^{*}}}{2 \cdot 2_{\mu}^{*}} \int_{\mathbb{R}^{N}} \int_{\mathbb{R}^{N}} \frac{\left|u_{\varepsilon}(x)\right|^{2_{\mu}^{*}}\left|u_{\varepsilon}(y)\right|^{2_{\mu}^{*}}}{|x-y|^{\mu}} d x d y\right\} \\
& +\frac{t_{\varepsilon}^{2}}{2} \int_{\mathbb{R}^{N}}\left|u_{\varepsilon}\right|^{2} d x-\int_{\mathbb{R}^{N}} F\left(t_{\varepsilon} u_{\varepsilon}\right) d x \\
\leq & \frac{N+2-\mu}{4 N-2 \mu} S_{H, L}^{\frac{2 N-\mu}{N-\mu+2}}+\frac{1}{2} d \varepsilon^{2}+O\left(\varepsilon^{N-2}\right)-\int_{B_{2 \rho}} F\left(t_{\varepsilon} u_{\varepsilon}\right) d x
\end{aligned}
$$

as $N \geq 5$. This implies that

$$
\Phi\left(t_{\varepsilon} u_{\varepsilon}\right) \leq \frac{N+2-\mu}{4 N-2 \mu} S_{H, L}^{\frac{2 N-\mu}{N-\mu+2}}+\frac{1}{2} d \varepsilon^{2}+O\left(\varepsilon^{N-2}\right)-\int_{B_{2 \rho}} F\left(t_{\varepsilon} u_{\varepsilon}\right) d x
$$

as $N \geq 5$. Similarly, we obtain

$$
\Phi\left(t_{\varepsilon} u_{\varepsilon}\right) \leq \frac{N+2-\mu}{4 N-2 \mu} S_{H, L}^{\frac{2 N-\mu}{N-\mu+2}}+\frac{1}{2} d \varepsilon^{2}|\log \varepsilon|+O\left(\varepsilon^{2}\right)-\int_{B_{2 \rho}} F\left(t_{\varepsilon} u_{\varepsilon}\right) d x
$$

as $N=4$.

By using $\left(f_{1}\right)-\left(f_{3}\right)$ and a similar proof to Lemma 3.5 in [9], we can get

$$
\lim _{\varepsilon \rightarrow 0^{+}} \varepsilon^{-2}|\log \varepsilon|^{-1} \int_{B_{2 \rho}} F\left(t_{\varepsilon} u \varepsilon_{\varepsilon}\right) d x=+\infty
$$


Thus, we have

$$
\sup _{t \geq 0} \Phi\left(t u_{0}\right)=\Phi\left(t_{\varepsilon} u_{\varepsilon}\right)<\frac{N+2-\mu}{4 N-2 \mu} S_{H, L}^{\frac{2 N-\mu}{N-\mu+2}}
$$

This completes the proof.

Proof of Theorem 2.2 From Lemmas 2.6 and 2.7 we can easily get the proof of Theorem 2.2.

\section{Proof of main results}

This section is devoted to proving our main results.

Proof of Theorem 1.1 Suppose that $\left\{u_{n}\right\}$ is a $(P S)_{c}$ sequence for $J$, that is,

$$
J\left(u_{n}\right) \rightarrow c \text { and } \quad J^{\prime}\left(u_{n}\right) \rightarrow 0 \quad \text { as } n \rightarrow \infty .
$$

Let $\tilde{a}(x)=a(x)-1$ and $\tilde{q}(x)=q(x)-1$, then

$$
\begin{aligned}
J\left(u_{n}\right)= & \frac{1}{2}\left\|u_{n}\right\|_{H^{1}\left(\mathbb{R}^{N}\right)}^{2}+\frac{1}{2} \int_{\mathbb{R}^{N}} \tilde{a}(x)\left|u_{n}\right|^{2} d x \\
& -\frac{1}{2 \cdot 2_{\mu}^{*}} \int_{\mathbb{R}^{N}} \int_{\mathbb{R}^{N}} \frac{\left|u_{n}(x)\right|^{2_{\mu}^{*}}\left|u_{n}(y)\right|^{2^{*}}}{|x-y|^{\mu}} d x d y \\
& -\frac{1}{p+1}\left\|u_{n}\right\|_{L^{p+1}\left(\mathbb{R}^{N)}\right.}^{p+1}-\frac{1}{p+1} \int_{\mathbb{R}^{N}} \tilde{q}(x)\left|u_{n}\right|^{p+1} d x \\
= & c+o(1)
\end{aligned}
$$

and

$$
\begin{aligned}
\left\langle J^{\prime}\left(u_{n}\right), u_{n}\right\rangle= & \left\|u_{n}\right\|_{H^{1}\left(\mathbb{R}^{N}\right)}^{2}+\int_{\mathbb{R}^{N}} \tilde{a}(x)\left|u_{n}\right|^{2} d x \\
& -\int_{\mathbb{R}^{N}} \int_{\mathbb{R}^{N}} \frac{\left|u_{n}(x)\right|^{2_{\mu}^{*}}\left|u_{n}(y)\right|^{2_{\mu}^{*}}}{|x-y|^{\mu}} d x d y \\
& -\left\|u_{n}\right\|_{L^{p+1}\left(\mathbb{R}^{N}\right)}^{p+1}-\int_{\mathbb{R}^{N}} \tilde{q}(x)\left|u_{n}\right|^{p+1} d x \\
= & \varepsilon_{n}\left\|u_{n}\right\|_{H^{1}\left(\mathbb{R}^{N}\right)},
\end{aligned}
$$

where $\varepsilon_{n} \rightarrow 0$ as $n \rightarrow \infty$. Observe that

$$
\begin{aligned}
& \left\|u_{n}\right\|_{H^{1}\left(\mathbb{R}^{N}\right)}, \quad \int_{\mathbb{R}^{N}} \tilde{a}(x)\left|u_{n}\right|^{2} d x, \\
& \int_{\mathbb{R}^{N}} \int_{\mathbb{R}^{N}} \frac{\left|u_{n}(x)\right|^{2_{\mu}^{*}}\left|u_{n}(y)\right|^{2 *}}{|x-y|^{\mu}} d x d y, \quad\left\|u_{n}\right\|_{L^{p+1}\left(\mathbb{R}^{N}\right)}^{p+1}
\end{aligned}
$$

are bounded, and thus we may assume

$$
u_{n} \rightarrow u^{0} \quad \text { weakly in } H^{1}\left(\mathbb{R}^{N}\right),
$$




$$
u_{n} \rightarrow u^{0} \quad \text { a.e. } \mathbb{R}^{N}
$$

So $u^{0}$ solves (1.1).

Define $v_{n}^{1}(x)=u_{n}(x)-u^{0}(x)$, we have

$$
\begin{aligned}
& v_{n}^{1} \rightarrow 0 \quad \text { weakly in } H^{1}\left(\mathbb{R}^{N}\right), \\
& \left\|v_{n}^{1}\right\|_{H^{1}\left(\mathbb{R}^{N)}\right.}^{2}=\left\|u_{n}\right\|_{H^{1}\left(\mathbb{R}^{N}\right)}^{2}-\left\|u^{0}\right\|_{H^{1}\left(\mathbb{R}^{N}\right)}^{2}+o(1), \\
& \left\|v_{n}^{1}\right\|_{L^{p+1}\left(\mathbb{R}^{N}\right)}^{p+1}=\left\|u_{n}\right\|_{L^{p+1}\left(\mathbb{R}^{N}\right)}^{p+1}-\left\|u^{0}\right\|_{L^{p+1}\left(\mathbb{R}^{N}\right)}^{p+1}+o(1), \\
& \int_{\mathbb{R}^{N}} \int_{\mathbb{R}^{N}} \frac{\left|v_{n}^{1}(x)\right|^{2_{\mu}^{*}}\left|v_{n}^{1}(y)\right|^{2_{\mu}^{*}}}{|x-y|^{\mu}} d x d y \\
& \quad=\int_{\mathbb{R}^{N}} \int_{\mathbb{R}^{N}} \frac{\left|u_{n}(x)\right|^{2_{\mu}^{*}}\left|u_{n}(y)\right|^{2_{\mu}^{*}}}{|x-y|^{\mu}} d x d y-\int_{\mathbb{R}^{N}} \int_{\mathbb{R}^{N}} \frac{\left|u^{0}(x)\right|^{2_{\mu}^{*}}\left|u^{0}(y)\right|^{2_{\mu}^{*}}}{|x-y|^{\mu}} d x d y+o(1) .
\end{aligned}
$$

Moreover, by (A2), we get

$$
\int_{\mathbb{R}^{N}} \tilde{a}^{ \pm}(x)\left|v_{n}^{1}\right|^{2} d x \rightarrow 0, \quad \int_{\mathbb{R}^{N}} \tilde{q}^{ \pm}(x)\left|v_{n}^{1}\right|^{2} d x \rightarrow 0 .
$$

It implies that

$$
J_{\infty}\left(v_{n}^{1}\right)=J\left(v_{n}^{1}\right)+o(1)=J\left(u_{n}\right)-J\left(u^{0}\right)+o(1)
$$

and

$$
J_{\infty}^{\prime}\left(v_{n}^{1}\right)=J^{\prime}\left(v_{n}^{1}\right)+o(1)=J^{\prime}\left(u_{n}\right)-J^{\prime}\left(u^{0}\right)+o(1) .
$$

Suppose $v_{n}^{1} \nrightarrow 00 \mathrm{n} H^{1}\left(\mathbb{R}^{N}\right)$, otherwise we are done. We claim that there is a sequence $\left\{y_{n}^{1}\right\} \subset \mathbb{R}^{N}$ such that $v_{n}^{1}\left(x+y_{n}^{1}\right) \rightarrow u^{1} \neq 0$ weakly in $H^{1}\left(\mathbb{R}^{N}\right)$.

First, we note that $J_{\infty}\left(v_{n}^{1}\right) \geq \alpha>0$. In fact, otherwise we would have

$$
\begin{aligned}
J_{\infty}\left(v_{n}^{1}\right)= & \frac{1}{2}\left\|v_{n}^{1}\right\|_{H^{1}\left(\mathbb{R}^{N}\right)}^{2}-\frac{1}{2 \cdot 2_{\mu}^{*}} \int_{\mathbb{R}^{N}} \int_{\mathbb{R}^{N}} \frac{\left|v_{n}^{1}(x)\right|^{2_{\mu}^{*}}\left|v_{n}^{1}(y)\right|^{2_{\mu}^{*}}}{|x-y|^{\mu}} d x d y \\
& -\frac{1}{p+1}\left\|v_{n}^{1}\right\|_{L^{p+1}\left(\mathbb{R}^{N}\right)}^{p+1} \\
\rightarrow & 0
\end{aligned}
$$

and

$$
\begin{aligned}
\left\langle J_{\infty}^{\prime}\left(v_{n}^{1}\right), v_{n}^{1}\right\rangle= & \left\|v_{n}^{1}\right\|_{H^{1}\left(\mathbb{R}^{N}\right)}^{2}-\int_{\mathbb{R}^{N}} \int_{\mathbb{R}^{N}} \frac{\left|v_{n}^{1}(x)\right|^{2_{\mu}^{*}}\left|v_{n}^{1}(y)\right|^{2_{\mu}^{*}}}{|x-y|^{\mu}} d x d y \\
& -\left\|v_{n}^{1}\right\|_{L^{p+1}\left(\mathbb{R}^{N}\right)}^{p+1} \\
= & \varepsilon_{n}\left\|u_{n}\right\|_{H^{1}\left(\mathbb{R}^{N}\right)} .
\end{aligned}
$$


These yield

$$
\begin{aligned}
\left(\frac{1}{2}\right. & \left.-\frac{1}{p+1}\right)\left\|v_{n}^{1}\right\|_{H^{1}\left(\mathbb{R}^{N}\right)}^{2} \\
& +\left(\frac{1}{p+1}-\frac{1}{2 \cdot 2_{\mu}^{*}}\right) \int_{\mathbb{R}^{N}} \int_{\mathbb{R}^{N}} \frac{\left|v_{n}^{1}(x)\right|^{2_{\mu}^{*}}\left|v_{n}^{1}(y)\right|^{2_{\mu}^{*}}}{|x-y|^{\mu}} d x d y \\
& \rightarrow 0,
\end{aligned}
$$

that is, $v_{n}^{1} \rightarrow 0 \mathrm{n} H^{1}\left(\mathbb{R}^{N}\right)$, a contradiction.

Set

$$
d_{n}=\left\|v_{n}^{1}\right\|_{L^{p+1}\left(\mathbb{R}^{N}\right)}^{p+1}+\int_{\mathbb{R}^{N}} \int_{\mathbb{R}^{N}} \frac{\left|v_{n}^{1}(x)\right|^{2_{\mu}^{*}}\left|v_{n}^{1}(y)\right|^{2_{\mu}^{*}}}{|x-y|^{\mu}} d x d y
$$

We claim that there is a $\beta>0$ independent of $n$ such that

$$
d_{n} \geq \beta>0 .
$$

Indeed, otherwise since $J_{\infty}^{\prime}\left(v_{n}^{1}\right) \rightarrow 0$, we have

$$
\begin{aligned}
J_{\infty}\left(v_{n}^{1}\right)= & \left(\frac{1}{2}-\frac{1}{2 \cdot 2_{\mu}^{*}}\right) \int_{\mathbb{R}^{N}} \int_{\mathbb{R}^{N}} \frac{\left|v_{n}^{1}(x)\right|^{2_{\mu}^{*}}\left|v_{n}^{1}(y)\right|^{2_{\mu}^{*}}}{|x-y|^{\mu}} d x d y \\
& +\left(\frac{1}{2}-\frac{1}{p+1}\right)\left\|v_{n}^{1}\right\|_{L^{p+1}\left(\mathbb{R}^{N}\right)}^{p+1}+o(1) \\
& \rightarrow 0
\end{aligned}
$$

this contradicts the fact $J_{\infty}\left(v_{n}^{1}\right) \geq \alpha>0$.

Now we decompose $\mathbb{R}^{N}$ into $N$-dimensional unit hypercubes $Q_{l}$ with vertices having integer coordinates. We distinguish two cases:

(i) $d_{n}^{1}=\max _{Q_{l}} \int_{Q_{l}}\left|v_{n}^{1}\right|^{p+1} d x \geq \beta>0$;

(ii) $\lim _{n \rightarrow \infty} \max _{Q_{l}} \int_{Q_{l}}\left|v_{n}^{1}\right|^{p+1} d x=0$, while

$$
d_{n}^{2}=\int_{\mathbb{R}^{N}} \int_{\mathbb{R}^{N}} \frac{\left|v_{n}^{1}(x)\right|^{2_{\mu}^{*}}\left|v_{n}^{1}(y)\right|^{2_{\mu}^{*}}}{\left.|x-y|\right|^{\mu}} d x d y \geq \beta>0
$$

In case (i), we denote by $y_{n}^{1}$ the center of a cube in which $d_{n}^{1}=\max _{Q_{l}} \int_{Q_{l}}\left|v_{n}^{1}\right|^{p+1} d x$. We can prove that $\left\{y_{n}^{1}\right\}$ is unbounded. Indeed, suppose by contradiction that $\left\{y_{n}^{1}\right\}$ is bounded, by passing to a subsequence, we find that $y_{n}^{1}$ would be in that same $Q_{l}$, and so they should coincide. In that $Q_{l}$, for some $n$ large, we have

$$
\left\|v_{n}^{1}\right\|_{H^{1}\left(Q_{l}\right)} \geq C\left\|v_{n}^{1}\right\|_{L^{p+1}\left(Q_{l}\right)} \geq \beta>0
$$

and

$$
\left.J_{\infty}\right|_{H^{1}\left(Q_{l}\right)}\left(\tilde{v}_{n}^{1}\right) \geq\left(\frac{1}{2}-\frac{1}{p+1}\right)\left\|v_{n}^{1}\right\|_{L^{p+1}\left(Q_{l}\right)}^{p+1} \geq C>0
$$




$$
\left.J_{\infty}^{\prime}\right|_{H^{1}\left(Q_{l}\right)}\left(\tilde{v}_{n}^{1}\right) \rightarrow 0 \quad \text { as } n \rightarrow \infty
$$

where

$$
\tilde{v}_{n}^{1}(x)= \begin{cases}v_{n}^{1}(x) & \text { for } x \in Q_{l}, \\ 0 & \text { for } x \in Q_{l}^{c}\end{cases}
$$

Hence, $v_{n}^{1}$ should converge strongly in $L^{p+1}\left(Q_{l}\right)$ to a nonzero function. This contradicts $v_{n}^{1} \rightarrow 0$ weakly in $H^{1}\left(\mathbb{R}^{N}\right)$. So $\left|y_{n}^{1}\right| \rightarrow+\infty$.

Let us recall $v_{n}^{1}\left(x+y_{n}^{1}\right) \rightarrow u^{1} \neq 0$ weakly in $H^{1}\left(\mathbb{R}^{N}\right)$. Arguing as before in the unit hypercube $Q$ center at the origin, we may conclude that $u^{1} \neq 0$. Moreover, $u^{1}$ solves (1.9). In the same way, let $v_{n}^{2}=v_{n}^{1}\left(x+y_{n}^{1}\right)-u^{1}$, and we may assume $v_{n}^{2} \rightarrow u^{2}$ weakly in $H^{1}\left(\mathbb{R}^{N}\right), u^{2}$ solves (1.9). Moreover,

$$
\begin{gathered}
\left\|v_{n}^{2}\right\|_{H^{1}\left(\mathbb{R}^{N)}\right)}^{2}=\left\|v_{n}^{1}\right\|_{H^{1}\left(\mathbb{R}^{N}\right)}^{2}-\left\|u^{1}\right\|_{H^{1}\left(\mathbb{R}^{N}\right)}^{2}+o(1) \\
=\left\|u_{n}\right\|_{H^{1}\left(\mathbb{R}^{N}\right)}^{2}-\left\|u^{0}\right\|_{H^{1}\left(\mathbb{R}^{N}\right)}^{2}-\left\|u^{1}\right\|_{H^{1}\left(\mathbb{R}^{N}\right)}^{2}+o(1), \\
J_{\infty}\left(v_{n}^{2}\right)=J_{\infty}\left(v_{n}^{1}\right)-J_{\infty}\left(u^{1}\right)+o(1) \\
=J_{\infty}\left(u_{n}\right)-J_{\infty}\left(u^{0}\right)-J_{\infty}\left(u^{1}\right)+o(1) .
\end{gathered}
$$

Iterating the above procedure, we obtain for a sequence of points $\left\{y_{n}^{j}\right\}$ and $v_{n}^{j}=v_{n}^{j-1}(x+$ $\left.y_{n}^{j-1}\right)-u^{j-1}$ that $v_{n}^{j} \rightarrow u^{j}$ weakly in $H^{1}\left(\mathbb{R}^{N}\right), u^{j}$ solves (1.9), and

$$
\begin{aligned}
& \left\|v_{n}^{j}\right\|_{H^{1}\left(\mathbb{R}^{N}\right)}^{2}=\left\|v_{n}^{j-1}\right\|_{H^{1}\left(\mathbb{R}^{N}\right)}^{2}-\left\|u^{j-1}\right\|_{H^{1}\left(\mathbb{R}^{N}\right)}^{2}+o(1), \\
& J_{\infty}\left(v_{n}^{j}\right)=J_{\infty}\left(v_{n}^{j-1}\right)-J_{\infty}\left(u^{j-1}\right)+o(1) .
\end{aligned}
$$

In case (ii), by the vanishing lemma of Lions, we have

$$
\lim _{n \rightarrow \infty}\left\|v_{n}^{1}\right\|_{H^{1}\left(\mathbb{R}^{N}\right)}^{p+1}=\lim _{n \rightarrow \infty}\left\|v_{n}^{1}\right\|_{H^{1}\left(\mathbb{R}^{N}\right)}^{2}=0 .
$$

Denote

$$
Q_{n}(r)=\sup _{x \in \mathbb{R}^{N}} \int_{B_{r}(x)}\left|\nabla v_{n}^{1}\right|^{2} d x,
$$

the concentration function of $v_{n}^{1}$. Choose $x_{n}^{1} \in \mathbb{R}^{N}$ and scale

$$
v_{n}^{1} \mapsto \tilde{v}_{n}^{1}(x)=R_{n}^{\frac{2-N}{2}} v_{n}^{1}\left(R_{n}^{-1}+x_{n}^{1}\right)
$$

such that

$$
\tilde{Q}_{n}(r)=\sup _{x, R_{n}^{-1}+x_{n}^{1} \in \mathbb{R}^{N}} \int_{B_{1}(x)}\left|\nabla \tilde{v}_{n}^{1}\right|^{2} d x=\int_{B_{1}(0)}\left|\nabla \tilde{v}_{n}^{1}\right|^{2} d x=\frac{1}{2 L} S_{H, L}^{\frac{N}{2}}
$$

for some $L>1$. Since $p<2^{*}-1$, we get

$$
\lim _{n \rightarrow \infty}\left\|\tilde{v}_{n}^{1}\right\|_{H^{1}\left(\mathbb{R}^{N}\right)}^{++1}=\lim _{n \rightarrow \infty}\left\|\tilde{v}_{n}^{1}\right\|_{H^{1}\left(\mathbb{R}^{N}\right)}^{2}=0 .
$$


Suppose $\tilde{v}_{n}^{1} \rightarrow v^{0}$ weakly in $H^{1}\left(\mathbb{R}^{N}\right), v^{0}$ is a solution of $(1.6)$, then we can show as $[6,31]$ that

$$
v_{n}^{2}=v_{n}^{1}-R_{n}^{\frac{N-2}{2}} v^{0}\left(R_{n}\left(x-x_{n}^{1}\right)\right)
$$

is a (PS) sequence of $I_{\infty}$, and we have

$$
\begin{gathered}
\left\|v_{n}^{2}\right\|_{H^{1}\left(\mathbb{R}^{N}\right)}^{2}=\left\|v_{n}^{1}\right\|_{H^{1}\left(\mathbb{R}^{N}\right)}^{2}-\left\|v^{0}\right\|_{H^{1}\left(\mathbb{R}^{N}\right)}^{2}+o(1) \\
=\left\|u_{n}\right\|_{H^{1}\left(\mathbb{R}^{N}\right)}^{2}-\left\|u^{0}\right\|_{H^{1}\left(\mathbb{R}^{N}\right)}^{2}-\left\|v^{0}\right\|_{H^{1}\left(\mathbb{R}^{N}\right)}^{2}+o(1) \\
J_{\infty}\left(v_{n}^{2}\right)=J_{\infty}\left(v_{n}^{1}\right)-I_{\infty}\left(v^{0}\right)+o(1) \\
=J_{\infty}\left(u_{n}\right)-J_{\infty}\left(u^{0}\right)-I_{\infty}\left(v^{0}\right)+o(1)
\end{gathered}
$$

Set $v_{n}^{j}=v_{n}^{j-1}-\bar{v}^{j-1}$, where $\bar{v}^{j-1}(x)=\left(R_{n}^{j}\right)^{\frac{N-2}{2}} v^{j}\left(R_{n}\left(x-x_{n}^{j}\right)\right)$ for sequences of points $x_{n}^{j}$ and $R_{n}^{j}$, Since $J_{\infty}\left(u^{j}\right) \geq c_{\infty}$ and $I_{\infty} \geq \frac{N+2-\mu}{4 N-2 \mu} S_{H, L}^{\frac{2 N-\mu}{N-2}}$, the iterations must stop after a finite number of times. The results follow easily.

Proof of Corollary 1.2 This is a direct consequence of Theorems 1.1 and 2.2, since the least energy of (1.6) is $\frac{N+2-\mu}{4 N-2 \mu} S_{H, L}^{\frac{2 N-\mu}{N-\mu+2}}$.

Proof of Corollary 1.3 It is standard to show that the energy $J$ has the mountain pass structure. Define

$$
c=\inf _{\gamma \in \Gamma} \sup _{t \in[0,1]} J(\gamma(t))
$$

where

$$
\Gamma=\left\{\gamma \in C\left([0,1], H^{1}\left(\mathbb{R}^{N}\right)\right) ; \gamma(0)=0, J(\gamma(1))<0\right\} .
$$

If we can prove $J$ satisfies the $(P S)_{c}$ condition, then $c$ is a nontrival critical value. To prove the $(P S)_{c}$ condition, it is sufficient to prove that $c<c_{\infty}$.

We note that $c_{\infty}$ is attained. In fact, by a similar argument to [20] (see also [4, 32]) via using the minimality property of the ground state to deduce some relationship between the function and its polarization, we can prove that the ground state solution of (1.9) has radial symmetry. Therefore, the Ekeland variational principle [10] and Lemma 2.5 imply $c_{\infty}$ is attained.

Let $w$ be the ground state solution of (1.9). Then $J(t w)>0$ for $t$ small and $J(t w) \rightarrow-\infty$ as $t \rightarrow \infty$. So there exists $t_{0}>0$ such that $J(t w)$ attains its maximum at $t_{0}$, then

$$
c \leq \max _{t>0} J(t w)=J\left(t_{0} w\right)<J_{\infty}\left(t_{0} w\right) \leq \max _{t>0} J_{\infty}(t w)=c_{\infty} .
$$

This completes the proof.

\section{Acknowledgements}

The authors would like to express their thanks to Prof. Jianfu Yang and Prof. Xiaohui Yu for their valuable comments and suggestions. 
Funding

This work is supported by the NSFC (Nos. 11701239 and 11871253), and by the NSF of Jiangxi province (20171BAB211003)

Availability of data and materials

Not applicable.

\section{Competing interests}

The authors declare that they have no competing interests.

\section{Authors' contributions}

All the authors contributed to each part of this study equally and approved the final version of the manuscript.

\section{Publisher's Note}

Springer Nature remains neutral with regard to jurisdictional claims in published maps and institutional affiliations.

Received: 15 April 2019 Accepted: 10 June 2019 Published online: 21 June 2019

\section{References}

1. Alves, C.O., Nóbrega, A.B., Yang, M.: Multi-bump solutions for Choquard equation with deepening potential well. Calc. Var. Partial Differ. Equ. 55(3), 48, 28 pp. (2016)

2. Alves, C.O., Yang, M.: Investigating the multiplicity and concentration behaviour of solutions for a quasi-linear Choquard equation via the penalization method. Proc. R. Soc. Edinb. A 146(1), 23-58 (2016)

3. Ambrosetti, A., Rabinowitz, P.H.: Dual variational methods in critical point theory and applications. J. Funct. Anal. 14, 349-381 (1973)

4. Bartsch, T., Weth, T., Willem, M.: Partial symmetry of least energy nodal solutions to some variational problems. J. Anal. Math. 96, 1-18 (2005)

5. Brézis, H., Lieb, E.: A relation between pointwise convergence of functions and convergence of functionals. Proc. Am. Math. Soc. 88, 486-490 (1983)

6. Cao, D., Peng, S.: A global compactness result for singular elliptic problems involving critical Sobolev exponent. Proc. Am. Math. Soc. 131, 1857-1866 (2003)

7. Cassani, D., Zhang, J.: Choquard-type equations with Hardy-Littlewood-Sobolev upper-critical growth. Adv. Nonlinear Anal. 8(1), 1184-1212 (2019)

8. Cingolani, S., Clapp, M., Secchi, S.: Multiple solutions to a magnetic nonlinear Choquard equation. Z. Angew. Math. Phys. 63, 233-248 (2012)

9. Deng, Y., Guo, Z., Wang, G.: Nodal solutions for p-Laplace equations with critical growth. Nonlinear Anal. 54(6), $1121-1151$ (2003)

10. Ekeland, I.: On the variational principle. J. Math. Anal. Appl. 17, 324-353 (1974)

11. Gao, F., Yang, M.: On the Brezis-Nirenberg type critical problem for nonlinear Choquard equaton. Sci. China Math. 61(7), 1219-1242 (2018). https://doi.org/10.1007/s11425-016-9067-5

12. Huang, Z., Yang, J., Yu, W.: Multiple nodal solutions of nonlinear Choquard equations. Electron. J. Differ. Equ. 2017, Paper No. 268, 18 pp. (2017)

13. Jin, L., Deng, Y.: A global compact result for a semilinear elliptic problem with Hardy potential and critical nonlinearities on $\mathbb{R}^{N}$. Sci. China Math. 53(2), 385-400 (2010)

14. Lieb, E.: Existence and uniqueness of the minimizing solution of Choquard's nonlinear equation. Stud. Appl. Math. 57 93-105 (1976/77)

15. Lieb, E., Loss, M.: Analysis, 2nd edn. Graduate Studies in Mathematics, vol. 14. Am. Math. Soc., Providence (2001)

16. Lions, P.L.: The Choquard equation and related questions. Nonlinear Anal. 4, 1063-1072 (1980)

17. Lions, P.L.: The concentration-compactness principle in the calculus of variations, the locally compact cases, part I. Ann. Inst. Henri Poincaré, Anal. Non Linéaire 1, 109-145 (1984)

18. Ma, L., Zhao, L.: Classification of positive solitary solutions of the nonlinear Choquard equation. Arch. Ration. Mech. Anal. 195, 455-467 (2010)

19. Ma, P., Zhang, J.: Existence and multiplicity of solutions for fractional Choquard equations. Nonlinear Anal. 164, 100-117 (2017)

20. Moroz, V., Schaftingen, J.: Groundstates of nonlinear Choquard equations: existence, qualitative properties and decay asymptotics. J. Funct. Anal. 265, 153-184 (2013)

21. Moroz, V., Schaftingen, J.: Existence of ground states for a class of nonlinear Choquard equations. Trans. Am. Math. Soc. 367, 6557-6579 (2015)

22. Moroz, V., Schaftingen, J.: Semi-classical states for the Choquard equation. Calc. Var. Partial Differ. Equ. 52, 199-235 (2015)

23. Moroz, V., Schaftingen, J.: Groundstates of nonlinear Choquard equations: Hardy-Littlewood-Sobolev critical exponent. Commun. Contemp. Math. 17, 1550005, 12 pp. (2015)

24. Papageorgiou, N.S., Rădulescu, V.D., Repovš, D.D.: Nonlinear Analysis-Theory and Methods. Springer Monographs in Mathematics. Springer, Cham (2019)

25. Pekar, S.: Untersuchungüber die Elektronentheorie der Kristalle. Akademie Verlag, Berlin (1954)

26. Penrose, R.: On gravity's role in quantum state reduction. Gen. Relativ. Gravit. 28, 581-600 (1996)

27. Pierrotti, D., Terracini, S.: On a Neumann problem with critical exponent and critical nonlinearity on the boundary. Commun. Partial Differ. Equ. 20,1155-1187 (1995)

28. Shen, Z., Gao, F., Yang, M.: Multiple solutions for nonhomogeneous Choquard equation involving Hardy-Littlewood-Sobolev critical exponent. Z. Angew. Math. Phys. 68(3), 61, 25 pp. (2017)

29. Singh, G.: Nonlocal perturbations of the fractional Choquard equation. Adv. Nonlinear Anal. 8(1), $694-706$ (2019) 
30. Strauss, W.: Existence of solitary waves in higher dimensions. Commun. Math. Phys. 55(2), 149-162 (1977)

31. Struwe, M.: Variational Methods. Applications to Nonlinear Partial Differential Equations and Hamiltonian Systems. Springer, Berlin (1990)

32. Van Schaftingen, J., Willem, M.: Symmetry of solutions of semilinear elliptic problems. J. Eur. Math. Soc. 10(2), 439-456 (2008)

33. Willem, M.: Functional Analysis, Fundamentals and Applications. Cornerstones, vol. XIV. Springer, New York (2013)

34. Ye, Y., Yu, X.: A global compactness result for a critical semilinear elliptic equation in $\mathbb{R}^{N}$. Nonlinear Anal. 71, 1844-1849 (2009)

Submit your manuscript to a SpringerOpen ${ }^{\odot}$ journal and benefit from:

- Convenient online submission

Rigorous peer review

- Open access: articles freely available online

- High visibility within the field

Retaining the copyright to your article

Submit your next manuscript at $\gg$ springeropen.com 